\title{
Musculoskeletal Medicine Is Underrepresented in the American Medical School Clinical Curriculum
}

\author{
Benedict F. DiGiovanni MD, Leigh T. Sundem BS, Richard D. Southgate MD, \\ David R. Lambert MD
}

Published online: 18 August 2015

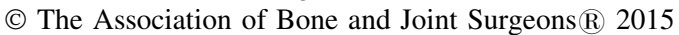

\begin{abstract}
Background Musculoskeletal (MSK) conditions are common, and their burden on the healthcare system is increasing as the general population ages. It is essential that medical students be well prepared to evaluate and treat MSK disorders in a confident manner as they enter the workforce. Recent studies and the American Association of Medical Colleges have raised concern that medical schools may not give sufficient instruction on this topic. Other authors have shown that preclinical instruction has increased over the past decade; however, it is unclear if required clinical instruction also has followed that trend.

Questions/purposes The purposes of this study were: (1) to assess the presence and duration of required or selective instruction in a MSK medicine specialty within the clinical years of undergraduate medical education; and (2) to assess the current state of requirements of clinical clerkships or rotations in other surgical and nonsurgical fields for comparison with the initial findings.
\end{abstract}

Each author certifies that he or she, or a member of his or her immediate family, has no funding or commercial associations (eg, consultancies, stock ownership, equity interest, patent/licensing arrangements, etc) that might pose a conflict of interest in connection with the submitted article.

All ICMJE Conflict of Interest Forms for authors and Clinical Orthopaedics and Related Research ${ }^{\circledR}$ editors and board members are on file with the publication and can be viewed on request.

B. F. DiGiovanni $(\bowtie)$, R. D. Southgate

Department of Orthopaedics and Rehabilitation, University of

Rochester Medical Center, 601 Elmwood Avenue, Box 665,

Rochester, NY 14642, USA

e-mail: Benedict_DiGiovanni@urmc.rochester.edu

L. T. Sundem, D. R. Lambert

Office of Medical Student Education, School of Medicine and

Dentistry, University of Rochester, Rochester, NY, USA
Methods The web sites of all 141 US medical schools were assessed to determine the content of their clinical curricula for the 2014-2015 academic year; five were excluded because they had not yet had a graduating class by the conclusion of the 2014-2015 academic year. Complete information on required rotations was obtained through the schools' web sites for all $136(100 \%)$ medical schools. For selective experience during the surgery clerkships, complete information was available for 130 of the remaining $136(96 \%)$ web sites.

Results Mean (in weeks, $\pm \mathrm{SD}$ ) duration of core clerkships were as follows: internal medicine $(10 \pm 2)$, surgery $(8 \pm 2)$, pediatrics $(7 \pm 1)$, obstetrics/gynecology $(6 \pm 1)$, and psychiatry $(5 \pm 1)$. Other common required clerkships were: family medicine (required in 96\% [131 of 136] of schools, mean duration of $6 \pm 2$ weeks), neurology ( $81 \%$ [110], $4 \pm 1$ ), and emergency medicine (55\% [75], $3 \pm 1$ ). Required MSK instruction, at a mean of $2 \pm 1$ weeks, was only present in $15 \%$ (20 of 136) of medical schools. In addition, clinical MSK instruction was offered as a selective (eg, students pick from a selection of subspecialties such as orthopaedics, plastics, or urology during a general surgery clerkship) in 34\% (44 of 130) of all medical schools. This is less than other non-core specialties: geriatrics/ambulatory care (required in 40\% [54 of 136] of schools, mean duration of $3 \pm 1$ weeks), critical care (30\% [41], mean of $3 \pm 1$ weeks), radiology (26\% [35], mean of $3 \pm 1$ weeks), anesthesiology (23\% [31], mean of $2 \pm 1$ weeks), and other surgical subspecialties (19\% [26], mean of $3 \pm 1$ weeks).

Conclusions Traditional core clerkships continue to be well represented in the clinical years, whereas three newer specialties have gained a larger presence: family medicine, neurology, and emergency medicine; these comprise the "big eight" of clinical clerkships. Given the high prevalence and burden of MSK disorders, required experience in MSK 
medicine continues to be underrepresented. Further discussion at a national level is needed to determine appropriate representation of MSK medicine specialties during the clinical years.

\section{Introduction}

Musculoskeletal (MSK) disorders are the leading cause of disability in the United States with approximately one in two American adults reporting a MSK condition that required medical care [19]. Approximately $20 \%$ of all visits to primary care clinics and emergency departments are the result of MSK complaints, second only to upper respiratory illness [7, 11, 13].

Even with this prevalence and subsequent burden on the healthcare system, medical school graduates may not be adequately equipped to diagnose and treat MSK conditions properly. Recent studies $[1,5,9,12,13,17,21]$ and the American Association of Medical Colleges (AAMC) [3] have raised concern that medical schools may not give sufficient instruction on this topic. In 2003, DiCaprio et al. [9] demonstrated the lack of required instruction in MSK medicine in both preclinical and clinical years of undergraduate medical education. The AAMC recommends that medical schools address these deficits by integrating a MSK medicine curriculum throughout the four years of medical school [3]. This allows graduating medical students to possess the skills to diagnose and appropriately initiate treatment for common MSK conditions as they would do if they were primary care providers. As a result of the AAMC initiative, increased emphasis has been placed on MSK instruction. Other authors [4] have shown that preclinical instruction has increased over the past decade; however, it is unclear whether required clinical instruction has also followed this trend.

Our primary aim was to assess the presence and duration of required or selective instruction in a MSK medicine specialty (orthopaedics, physical medicine and rehabilitation $[\mathrm{PM} \& \mathrm{R}]$, rheumatology, or primary care sports medicine) within the clinical years of undergraduate medical education. Our secondary aim was to assess the current state of requirements of clinical clerkships or rotations in other surgical subspecialties (urology, neurosurgery, vascular surgery, plastic surgery, otolaryngology, ophthalmology, cardiac surgery) and nonsurgical fields for comparison with the initial findings.

\section{Materials and Methods}

All 141 accredited medicals schools in the United States for the 2014-2015 academic year were identified through the medical school directory on the AAMC web site [2]. Medical schools were excluded if they had not yet had a graduating class by the conclusion of the 2014-2015 academic year $(\mathrm{n}=5)$, leaving a total of 136 medical schools for analysis. Complete curriculum information for required clinical clerkships was obtained through the web sites for all $136(100 \%)$ medical schools. Web sites were most recently updated in 2014-2015 for 127 of 136 (93\%) schools; nine schools (7\%) had their web sites updated in 2013 or prior. For medical schools $(n=35$ [26\%]) with unavailable or insufficient data regarding the rotations during the surgery clerkship or selective offerings, a phone call to the clerkship coordinator was made to gather the necessary information. There were complete data regarding the specifics of the surgery clerkship for 130 schools (130 of 136 [96\%]) at the final analysis.

For the acquisition of curriculum data, a standardized method roughly approximating the method used by DiCaprio et al. [9] was used. The presence and duration of a clinical clerkship in MSK medicine (orthopaedics, PM\&R, rheumatology, and primary care sports medicine) as well as the presence of a required rotation in orthopaedics during the required surgical clerkship were determined. The option of a selective, defined as a rotation that is required but with the freedom to choose the service to rotate on out of a variety of options of specialty services (such as the option to rotate on the orthopaedics, neurosurgery, or plastics service during the required general surgery clerkship or cardiology, oncology, or nephrology service during the internal medicine clerkship), in these specialties was also assessed. Other information gathered from the web sites included identification of required clinical clerkships and rotations in all other specialties, surgical and nonsurgical, at each school along with the duration. The surgical subspecialties included urology, neurosurgery, vascular surgery, plastic surgery, otolaryngology, ophthalmology, and cardiac surgery.

\section{Results}

Core Clerkships and Other Required Clinical Rotations

The core clerkships, comprised of five different specialties, were required in all medical schools. Mean duration (in weeks, $\pm \mathrm{SD}$ ) of these core clerkships were as follows: internal medicine (10 \pm 2 weeks), surgery ( $8 \pm 2$ weeks), pediatrics ( $7 \pm 1$ weeks), obstetrics/gynecology ( $6 \pm 1$ weeks), and psychiatry ( $5 \pm 1$ weeks). Other common required clerkships were: family medicine (required in $96 \%$ [131 of 136] of schools, mean duration of $6 \pm 2$ weeks), emergency medicine (55\% [75], $3 \pm 1$ weeks), and neurology (81\% [110], $4 \pm 1$ weeks) (Table 1). 
Table 1. Other required clinical rotations*

\begin{tabular}{|c|c|c|c|c|c|c|c|c|c|}
\hline $\begin{array}{l}\text { Specifications of schools } \\
\text { with regard to clerkships }\end{array}$ & $\begin{array}{l}\text { Family } \\
\text { medicine }\end{array}$ & Neurology & $\begin{array}{l}\text { Emergency } \\
\text { medicine }\end{array}$ & $\begin{array}{l}\text { Intensive } \\
\text { care unit }\end{array}$ & Radiology & Anesthesia & Other & $\begin{array}{l}\text { Other surgical } \\
\text { subspecialty }\end{array}$ & MSK \\
\hline chools require, number $(\%)$ & $\begin{array}{l}131 \\
(96 \%)\end{array}$ & $\begin{array}{l}110 \\
(81 \%)\end{array}$ & $\begin{array}{l}75 \\
(55 \%)\end{array}$ & $\begin{array}{l}41 \\
(30 \%)\end{array}$ & $\begin{array}{l}35 \\
(26 \%)\end{array}$ & $\begin{array}{l}31 \\
(23 \%)\end{array}$ & $\begin{array}{l}54 \\
(40 \%)\end{array}$ & $\begin{array}{l}26 \\
(19 \%)\end{array}$ & $\begin{array}{l}20 \\
(15 \%)\end{array}$ \\
\hline Duration \pm SD (weeks) & $6 \pm 2$ & $4 \pm 1$ & $3 \pm 1$ & $3 \pm 1$ & $3 \pm 1$ & $2 \pm 1$ & $3 \pm 1$ & $3 \pm 1$ & $2 \pm 1$ \\
\hline
\end{tabular}

* All percentages listed are number of schools requiring a rotation in the listed specialty out of 136 total medical schools.

Other = geriatrics/ambulatory care; MSK = musculoskeletal.

Table 2. MSK clinical curriculum

\begin{tabular}{|c|c|c|c|c|c|c|}
\hline \multirow{2}{*}{$\begin{array}{l}\text { Specifications of schools } \\
\text { with regard to clerkships }\end{array}$} & \multirow{2}{*}{$\begin{array}{l}\text { Total required } \\
\text { MSK clinical } \\
\text { exposure }\end{array}$} & \multicolumn{2}{|c|}{ Required standalone rotations: MSK medicine } & \multicolumn{3}{|c|}{ Required rotations during surgery clerkship } \\
\hline & & Orthopaedics & $\begin{array}{l}\text { Other: } \mathrm{PM} \& \mathrm{R} \text {, rheumatology, } \\
\text { primary care sports medicine }\end{array}$ & Orthopaedics & $\begin{array}{l}\text { Other surgical } \\
\text { subspecialty }\end{array}$ & Other \\
\hline Schools have, number $(\%)$ & $\begin{array}{l}20 / 136 \\
(15 \%)\end{array}$ & $\begin{array}{l}8 / 136 \\
(6 \%)\end{array}$ & $\begin{array}{l}8 / 136 \\
(5 \%)\end{array}$ & $\begin{array}{l}6 / 130 \\
(5 \%)\end{array}$ & $\begin{array}{l}17 / 130 \\
(13 \%)\end{array}$ & $\begin{array}{l}10 / 130 \\
(8 \%)\end{array}$ \\
\hline $\begin{array}{l}\text { Average duration if } \\
\text { offered } \pm \text { SD (weeks) }\end{array}$ & $2 \pm 1$ & $2 \pm 1$ & $2 \pm 1$ & - & - & - \\
\hline
\end{tabular}

Other required rotation during surgery clerkship = anesthesiology, emergency medicine, cardiothoracic surgery, ophthalmology, otolaryngology, plastic surgery, vascular surgery, urology; MSK = musculoskeletal; PM\&R = physical medicine and rehabilitation.

\section{Musculoskeletal Clinical Curriculum}

Required MSK clinical exposure is summarized in Table 2). Total required MSK clinical instruction, averaging $2 \pm 1$ weeks, was present in only $15 \%$ (20 of 136) of medical schools. Required standalone MSK clinical rotations and required rotations during surgical clerkship are further stratified (Table 2). This was notably shorter than other non-core specialties: geriatrics/ambulatory care (required in 40\% [54 of 136] of schools, mean duration of $3 \pm$ 1 weeks), critical care (30\% [41], $3 \pm 1$ weeks), and radiology (26\% [35], $3 \pm 1$ weeks). Only anesthesiology (23\% [31], $2 \pm 1$ weeks) and other surgical subspecialties (19\% [26], $3 \pm 1$ weeks) were comparable in terms of duration (Fig. 1) or required instruction (Fig. 2). MSK medicine was offered as a standalone selective in 35\% (47 of 136) and surgical selective (as a part of the general surgery clerkship) in 34\% (44 of 130) of all medical schools.

Of the required MSK medicine clinical rotations, an orthopaedics rotation was required in eight medical schools (6\% [eight of 136]) as a clerkship and in six schools (5\% [six of 136]) as part of the surgery clerkship. PM\&R was required in seven schools (5\% [seven of 136]) and rheumatology only required in one medical school $(<1 \%$ [one of 136]). Of these medical schools with required MSK clinical rotations, one school $(<1 \%$ [one of 136]) required rotations in all three and was therefore counted only once in the final tally to total 20 medical schools with required
MSK clinical instruction. Primary care sports medicine was not a required rotation in any medical school.

\section{Discussion}

Musculoskeletal conditions are common, and their economic impact in terms of healthcare costs and lost wages totaled $\$ 950$ billion (USD), or $7 \%$ of the gross domestic product in 2006 [19]. In 2005, AAMC raised concern that medical schools do not provide sufficient instruction on the topic. Prior studies have demonstrated an increase in preclinical instruction $[4,9]$ but it is unclear whether there is a similar trend in clinical MSK instruction. The current study sought to assess the presence and duration of required or selective clinical instruction in MSK medicine. We found that a rotation in MSK medicine is currently required in only 20 of the medical schools (15\%), making it the least represented of all required specialties. In other words, $85 \%$ (116 of 136) of all US medical schools do not require a clinical rotation in MSK medicine. Requirements include rotations in orthopaedics in 14 schools, PM\&R in seven schools, and rheumatology in one school with overlap at one school that required all three. MSK medicine, when required, also represented the shortest duration (less than $2 \pm 1$ weeks) of required instruction of all the required rotations.

There are limitations of this study which should be considered. The current study only assessed clinical instruction in MSK medicine without concurrent assessment of the 


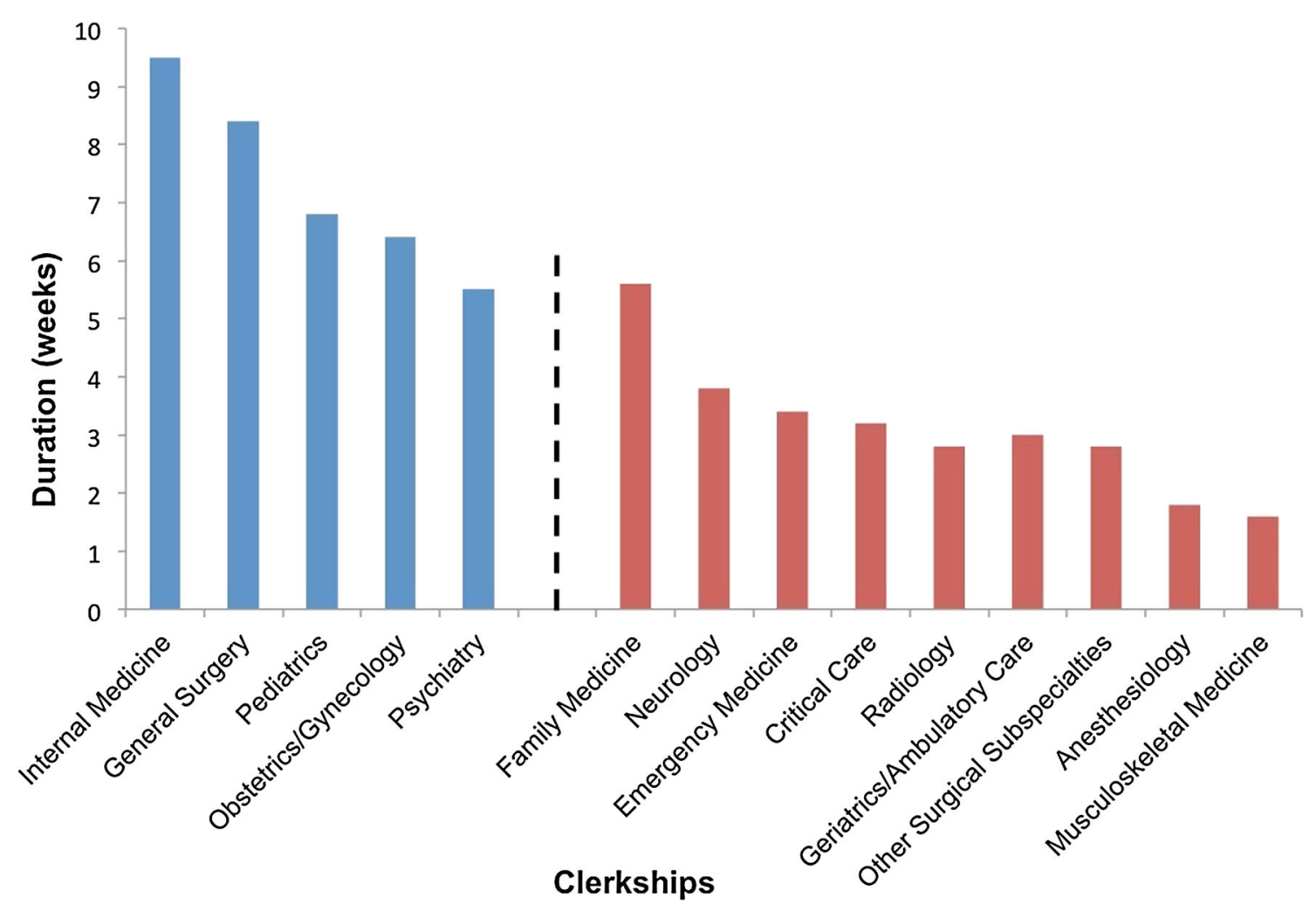

Fig. 1 All core clerkships (blue bars) and non-core clerkships (red bars) required during the clinical curriculum in medical school with their respective durations of instruction are shown. Each

preclinical coursework. With findings from a prior study suggesting a significant majority of medical schools are now formally teaching the MSK system in the preclinical years [4], we acknowledge and are encouraged to see that most students in US medical schools are receiving instruction in the basic science of the MSK system. We also did not address clinical instruction on MSK disorders that may occur during other required rotations. Students will likely be exposed to patients with MSK conditions while on family medicine, internal medicine, and emergency medicine and may receive some lectures on the topic. However, these rotations do not typically have a substantial amount of time devoted to MSK clinical problems and exposure to MSK disorders is likely highly variable and incomplete. Along those lines, we have noted that approximately one-third of schools offer a selective in MSK medicine but it is beyond the scope of this study to ascertain participation in these courses. Lastly, there may be potential for error associated with using web sites for data collection, because some web sites may be more comprehensive and up to date than others. However, we believe this methodology was the most appropriate to complete a thorough review of curricular information that also minimized potential for response bias. clinical rotation is separated by specialty and graphed to show the mean duration (in weeks) for each of the required rotations represented.

In 2003, DiCaprio et al. [9] originally reported the status of MSK medicine instruction within the medical school curriculum and found only $21 \%$ of medical schools required clinical experience in MSK medicine at that time. These findings corresponded with the state of MSK medical education at the beginning of the US Bone and Joint Decade (2002-2011), during which time the World Health Organization, AAMC, and the American Academy of Orthopaedic Surgeons promoted MSK medicine education in an effort to address these curricular deficiencies. In a followup study, Bernstein and colleagues [4] reported that by $2009,79 \%$ of American medical schools had incorporated a required MSK course or block in the preclinical years, up from $42 \%$ in 2003 [9]. However, no major improvement was seen in the clinical curriculum, with only $24 \%$ of medical schools requiring clinical experience in MSK medicine [4], a small increase from the $21 \%$ in 2003 [9]. Our current findings indicate that MSK medicine continues to be underrepresented in the 2014-2015 undergraduate medical school clinical curriculum with a required clinical rotation in a MSK medicine specialty only seen in $15 \%$ of all medical schools. It should be noted that there are differences in the methodology for obtaining information when comparing our data with 2009 data [4]. 


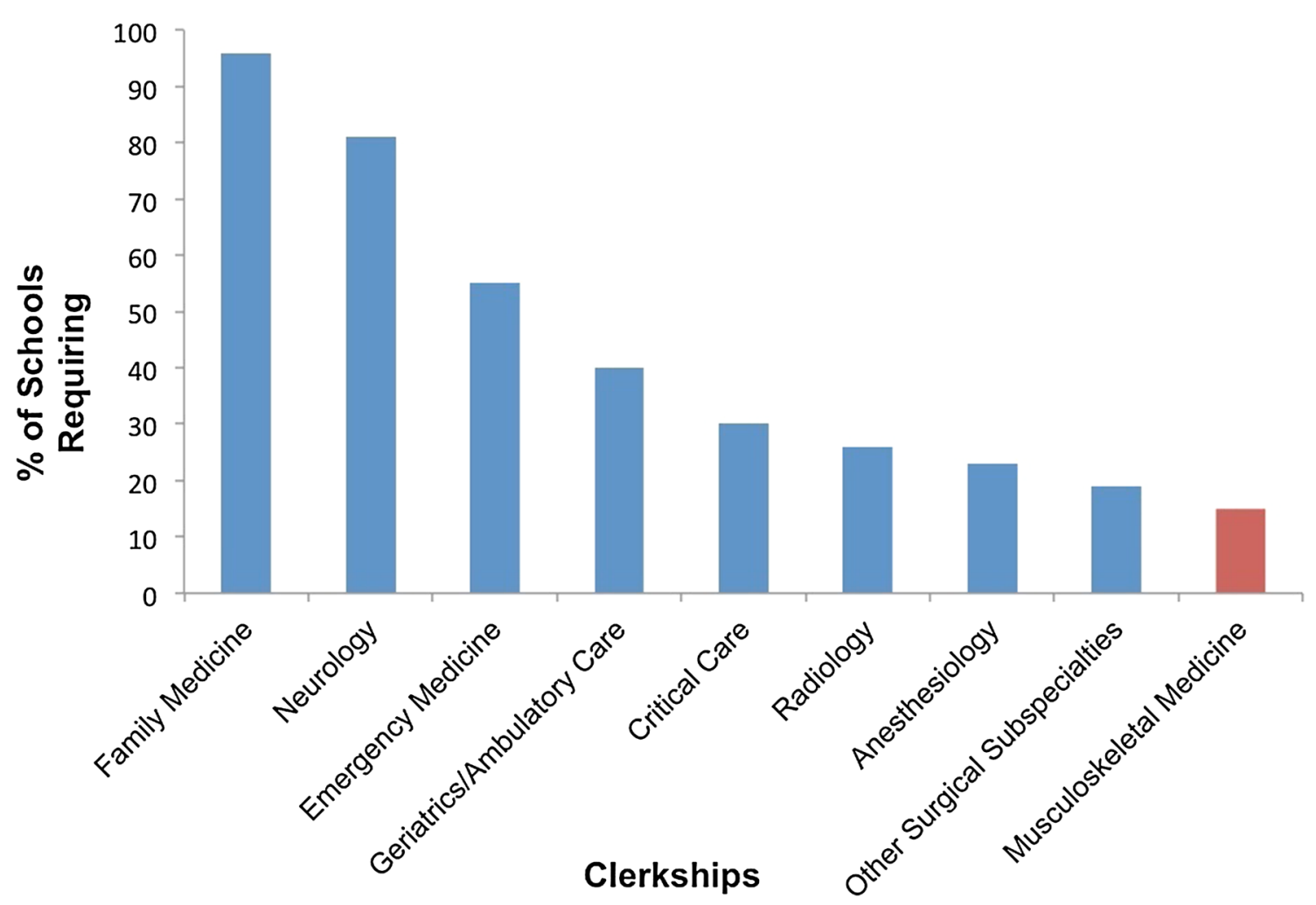

Fig. 2 Required clinical rotations in non-core specialties currently present in medical schools are shown with the relative frequency of each rotation. Each specialty is represented by the percentage of

That study [4] obtained information by email correspondence to the dean of students at each medical school. We chose to use a more systematic and objective method of data collection by review of each school's web site with only a few schools having incomplete information in regard to details within the required surgery core clerkship. Although different methodologies were used, these studies each highlight what appears to be little or no advancement in the required exposure to MSK clinical medicine from the beginning of the Bone and Joint Decade until the present time.

The traditional core clerkships (internal medicine, surgery, pediatrics, gynecology, psychiatry) continue to be required at all medical schools, whereas some other specialties are now becoming heavily represented. Family medicine can now be considered a core clerkship in almost all medical schools (required in 96\% [131 of 136]). Neurology ( $81 \%$ [110 of 136]) and emergency medicine (55\% [75 of 136]) are becoming mainstays in the curriculum with over half of all schools requiring clinical experience in these disciplines. These three newer specialties have gained a stronger presence in American medical schools' clinical curricula. Along with the five traditional core clerkships, they can be thought of as the "big eight" clerkships, which medical students commonly complete during their clinical medical schools that require a rotation in that specialty out of the total number of American medical schools included in the study $(n=$ 136).

years. Even specialized fields such as critical care, radiology, and anesthesiology are gaining headway in becoming mandatory rotations with approximately $25 \%$ of schools requiring exposure for their students. This evolution of clinical training is a positive development in medical education because medical students are now receiving a more comprehensive educational experience with exposure in many different specialty fields, representative of the diversity of medical problems in $21^{\text {st }}$ century medicine. Unfortunately, MSK medicine specialties lack a similar presence within the clinical years with $85 \%$ (116 of 136) of schools having no required rotation in these specialties. Within the surgical fields, orthopaedics is the least represented, especially when compared with the requirements for other surgical subspecialties both as a required clerkship (6\% versus $19 \%)$ and as a rotation within the general surgery clerkship (5\% versus $13 \%$ ).

Underrepresentation of MSK medicine in the clinical curriculum of US medical schools may be detrimental to overall quality of patient care given the high prevalence of MSK disease in the American population. The discrepancy is highlighted when comparing the representation of a topic in the medical school curriculum with its prevalence in primary care and urgent care settings. Approximately $20 \%$ of all visits to primary care clinics and emergency 
departments are the result of MSK complaints, second only to upper respiratory illness $[7,11,13]$.

A number of studies have found that inadequate clinical exposure to MSK medicine during medical school could result in residents and physicians across many specialties who feel underprepared to appropriately evaluate MSK conditions $[1,5,6,11,12,15]$. For the majority of physicians entering general practice, the primary instruction they receive in the diagnosis and initial management of MSK disorders is during their medical school education [18] because most residents in non-MSK specialties are provided little additional instruction in MSK medicine during their residency training $[11,14,20]$. These findings highlight that the absence of required clinical experience in MSK medicine may have negative consequences for providers in their clinical practice. Addressing the disproportionately limited clinical exposure to MSK medicine and related disorders during medical school would likely provide great benefit to graduates in their future role as independent clinicians. This potential has been recognized by the AAMC, which recommended that medical schools identify where MSK medicine is currently taught within the four year medical education and either increase instruction time or improve the integration of MSK medicine into the entirety of the curriculum [3].

In a prior study by the current authors evaluating the factors that impact the MSK knowledge and clinical confidence of graduating medical students, it was noted that the only factor that led to a significant increase in MSK medical knowledge exposure to an MSK elective [10]. Only two weeks were required to significantly increase knowledge; however, no concomitant improvement was observed in clinical confidence. Even rotations as long as five weeks failed to result in clear improvements in clinical confidence. This indicates that merely participating in an elective in orthopaedic surgery or spending additional time during the elective experience may not be sufficient for obtaining the appropriate clinical readiness to evaluate basic MSK conditions. Ideal clinical MSK instruction may be multidisciplinary with students being exposed to a formal curriculum with clinical instruction by orthopaedists, physiatrists (PM\&R), rheumatologists, and primary care sports medicine specialists. Another option suggested by educators is the formal integration of this topic throughout the clinical years with incorporation of dedicated instruction and clinical experiences in MSK conditions within core clerkships such as internal and emergency medicine and family practice $[8,16]$.

During the last decade, great strides have been made in terms of MSK instruction in the preclinical curriculum in that now there is dedicated preclinical instruction in the MSK system in almost all medical schools. However, the current study highlights that important deficiencies remain within the clinical education of medical students in the evaluation and initiation of treatment for common MSK conditions, with $85 \%$ (116 of 136) of US medical schools lacking required clinical MSK instruction. The presence and duration of required clinical MSK instruction are lower than that for all the other specialties that were reported in this study, including non-core specialties such as emergency medicine, critical care, and radiology, which does not reflect the substantial burden of MSK conditions on the American population. Possible solutions include the addition of a required interdisciplinary clinical rotation in MSK medicine or the integration of instruction in MSK conditions throughout the clinical years within the currently required core clerkships. Further discussion at a national level is needed to determine appropriate representation of MSK medicine specialties during the clinical years and how to implement the necessary curricular reform to address these deficiencies.

\section{References}

1. Al-Nammari SS, Pengas I, Asopa V, Jawad A, Rafferty M, Ramachandran M. The inadequacy of musculoskeletal knowledge in graduating medical students in the United Kingdom. $J$ Bone Joint Surg Am. 2015;97:e36.

2. Association of American Medical Colleges. Available at: https:// members.aamc.org/eweb/DynamicPage.aspx? site=AAMC\&web code $=$ AAMCOrgSearchResult \&orgtype=MedicalSchool. Accessed August 15, 2014.

3. Association of American Medical Colleges. Contemporary Issues in Medicine: Musculoskeletal Medicine Education. Washington, DC, USA: AAMC; 2005.

4. Bernstein J, Garcia GH, Guevara JL, Mitchell GW. Progress report: the prevalence of required medical school instruction in musculoskeletal medicine at decade's end. Clin Orthop Relat Res. 2011;469:895-897.

5. Clawson DK, Jackson DW, Ostergaard DJ. It's past time to reform the musculoskeletal curriculum. Acad Med. 2001;76:709710 .

6. Comer GC, Liang E, Bishop JA. Lack of proficiency in musculoskeletal medicine among emergency medicine physicians. J Orthop Trauma. 2014;28:e85-87.

7. Craton N, Matheson GO. Training and clinical competency in musculoskeletal medicine. Identifying the problem. Sports Med. 1993;15:328-337.

8. Day CS, Yeh AC, Franko O, Ramirez M, Krupat E. Musculoskeletal medicine: an assessment of the attitudes and knowledge of medical students at Harvard Medical School. Acad Med. 2007;82:452-457.

9. DiCaprio MR, Covey A, Bernstein J. Curricular requirements for musculoskeletal medicine in American medical schools. $J$ Bone Joint Surg Am. 2003;85:565-567.

10. DiGiovanni BF, Southgate RD, Mooney CJ, Chu JY, Lambert DR, O'Keefe RJ. Factors impacting musculoskeletal knowledge and clinical confidence in graduating medical students. $J$ Bone Joint Surg Am. 2014;96:e185.

11. Freedman KB, Bernstein J. The adequacy of medical school education in musculoskeletal medicine. J Bone Joint Surg Am. 1998;80:1421-1427. 
12. Freedman KB, Bernstein J. Educational deficiencies in musculoskeletal medicine. J Bone Joint Surg Am. 2002;84:604-608.

13. Grunfeld R, Banks S, Fox E, Levy BA, Craig C, Black K. An assessment of musculoskeletal knowledge in graduating medical and physician assistant students and implications for musculoskeletal care providers. J Bone Joint Surg Am. 2012;94: 343-348.

14. Matheny JM, Brinker MR, Elliott MN, Blake R, Rowane MP. Confidence of graduating family practice residents in their management of musculoskeletal conditions. Am J Orthop (Belle Mead NJ). 2000;29:945-952.

15. Matzkin E, Smith EL, Freccero D, Richardson AB. Adequacy of education in musculoskeletal medicine. J Bone Joint Surg Am. 2005;87:310-314.

16. Schmale GA. More evidence of educational inadequacies in musculoskeletal medicine. Clin Orthop Relat Res. 2005;437:251-259.
17. Skelley NW, Tanaka MJ, Skelley LM, LaPorte DM. Medical student musculoskeletal education: an institutional survey. $J$ Bone Joint Surg Am. 2012;94:e146(1-7).

18. Sneiderman C. Orthopedic practice and training of family physicians: a survey of 302 North Carolina practitioners. J Fam Pract. 1977;4:267-270.

19. United States Bone and Joint Initiative. The Burden of Musculoskeletal Diseases in the United States. Rosemont, IL, USA: American Academy of Orthopaedic Surgeons; 2011.

20. Williams JR. The teaching of trauma and orthopaedic surgery to the undergraduate in the United Kingdom. J Bone Joint Surg Br. 2000;82:627-628.

21. Yeh AC, Franko O, Day CS. Impact of clinical electives and residency interest on medical students' education in musculoskeletal medicine. J Bone Joint Surg Am. 2008;90:307-315. 